\title{
Laparoscopic single port surgery nephrectomy in a child - initial experience
}

\author{
Anna Bryks-Laszkowska, Andrzej Gołębiewski, Piotr Czauderna \\ Department of Surgery and Urology for Children and Adolescents, Medical University of Gdansk, Poland
}

Videosurgery Miniinv 2012; 7 (4): 304-306 DOI: 10.5114/wiitm.2011.30515

\begin{abstract}
The aim of the stucdy was to present technical aspects and possible intraoperative complications based on the first single port surgery (SPS) laparoscopic nephrectomy performed in our center. The SPS laparoscopic nephrectomy of the right kidney was performed using a transperitoneal access in a 4-year-old child due to a small nonfunctional kidney complicated by hypertension. The intraoperative course was complicated by rupture of the gall bladder wall after grasper removal without leakage of its contents into the peritoneal cavity. No postoperative complications occurred. The SPS nephrectomy appears to be an advantageous method in children which offers a good cosmetic effect. Other benefits require confirmation in further studies. Fixation of the gall bladder is a very helpful but risky maneuver.
\end{abstract}

Key words: nephrectomy, single port surgery technique, laparoscopy.

\section{Introduction}

In the pursuit of minimized tissue injury resulting in decreased postoperative pain and increased emphasis on the cosmetic effect, single port laparoscopic procedures (SPS) have gained in popularity, even though they are technically more challenging and require longer training in comparison to traditional laparoscopy [1-3]. Increasing interest in this branch of minimally invasive surgery is also fuelled by huge technological advancement. The use of SPS in pediatric surgery is still more restricted than in adults. The indications for the method in children, similarly to adults, are not clearly defined. Publications concerning single incision laparoscopic surgery (SILS) technique in children are still very few [4-8].

The aim of the study was to present technical aspects and possible intraoperative complications based on the first single port surgery (SPS) laparoscopic nephrectomy performed in our center.

\section{Case report}

One nephrectomy was performed in our center during 18 months from 2009 to 2011 among all 20 SPS procedures. A 4-year-old child was selected for nephrectomy due to a small nonfunctional right kidney causing arterial hypertension requiring pharmacotherapy. Ultrasound imaging revealed a small, aplastic right kidney sized $29 \mathrm{~mm} \times 14 \mathrm{~mm} \times 14 \mathrm{~mm}$. Functional examination (EC dynamic scintigraphy with a furosemide test) showed no secretory function in the right kidney. The child was operated on through a transperitoneal approach, in a supine position. A 3-channel GelPort (Applied Medical) was inserted through a $2 \mathrm{~cm}$ minilaparotomy wound. One channel was used to insert the $5 \mathrm{~mm} 30^{\circ}$ optic. Insuf- 
flation at $10 \mathrm{~mm} \mathrm{Hg}$ was created. Reusable, straight instruments were used for the procedure. A disposable needle grasper manufactured by Stryker was placed through the $2 \mathrm{~mm}$ GelPort in order to affix the gallbladder and expose the kidney, which is why the procedure fulfilled the SPS criteria. The kidney was dissected together with the ureter without complications using a monopolar hook. The renal vascular pedicle and ureter were coagulated by the LigaSure device. The kidney was removed through the navel jacketed with the sleeve of the GelPort. Although, towards the end of the procedure during the removal of the grasper, which affixed the fundus of the gallbladder, its wall was torn, no contents leaked into the peritoneum. That is why after minimal laparoscopic mobilization of the gallbladder fundus, it was exposed through the umbilical incision (without widening) and closed with absorbable sutures in two layers. The postoperative course was uncomplicated. SPS nephrectomy lasted for $120 \mathrm{~min}$ and no complications other than tearing of the gallbladder wall occurred. The blood loss was minimal. The postoperative course was uncomplicated and the length of hospital stay was 3 days. The child was administered paracetamol only and pethidine in a single dose, directly after the procedure. The healing of the postoperative wound was normal. The cosmetic result of the procedure was assessed as very good during the follow-up appointment. An ultrasound performed 3 days after the procedure did not reveal any abnormalities in the kidney site and the gallbladder, except for the thickened wall around its fundus.

\section{Discussion}

Recently, a slow, but visible trend in pediatric surgery towards miniinvasive techniques, conventional laparoscopy being the most often performed, has taken place. Single port surgery (SPS) is still rarely performed but is gaining in popularity $[1,9]$. Nevertheless, the number of publications concerning SPS in children is considerably lower than the number of reports concerning adults. The difference is in accordance with usual practice when new surgical techniques are introduced in the case of children. The introduction of a new surgical procedure in children requires, most of all, adjustment of the size of surgical instruments. Miniaturization of equipment and its adaptation for pediatric surgery involves an increase of the procedure costs and delays the availability of the newest surgical procedures $[4,10]$. Benefits of SPS technique are still arguable even in grownups. A very good cosmetic result is believed to be the main advantage in comparison to classic laparoscopy [3, 4, 8, 11]. Numerous publications report other theoretical benefits such as decrease of postoperative pain, decrease of blood loss, shorter hospital stay, and also reduced risk of organ damage connected with the necessity of additional ports placement, which results in cost reduction $[1,4,5,9,12]$. Appropriate choice of patients is one of the most crucial factors influencing the SPS results and minimizing the number of conversions to classic laparoscopy [1]. According to Kaouk et al., the proportion of conversions to classic laparoscopy in a trial including 1076 cases was about $20 \%$ [1], which appears to be a promising result given the very early stage of the method's development. The range of indications for SPS is increasing quite dynamically and includes, besides common resective procedures such as nephrectomy, cholecystectomy and adrenalectomy, oncological procedures and harvesting kidney for transplantation [1].

Among SPS procedures, reconstructive procedures are outnumbered by resective procedures. It results from greater technical difficulties connected with the limited area of the surgical field constricting precise maneuvers and the necessity to suture, which is very hard in SPS technique [1,12]. The most common in SPS is the umbilical approach, used in $70 \%$ of SILS operations [1]. Considering safety and comfort, nephrectomy using the transperitoneal approach appears to be the most beneficial choice due to cosmetic results, larger surgical field and easier access to the organ, especially in small children [8]. Intraoperative complications occur in $3-4 \%$ of cases described in the literature, and vascular damage predominates. Organ injuries constitute only $1 \%$ of cases in published studies. Intraoperative damage of the gallbladder is an extremely rare complication $[1,5]$. Gallbladder fixation in order to achieve better visualization of the right kidney, in the case described, is very beneficial, but entails the risk of organ injury, although it may have also resulted from a technical mistake. Nevertheless, $2 \mathrm{~mm}$ needle instruments may be helpful in SPS procedures, as they can be placed via both the same working port without an additional trocar and percutaneous puncture directly next to the area operated on [2]. 
The SPS technique is difficult due to collision of instruments with optics, limited triangulation and the small surgical field, which makes maneuvering during the procedure in a pediatric patient more challenging. Limited access to ports and instruments adapted to the patient's size and their high price are substantial factors hampering the advancement of the technique. However, reports showing the employment of classic laparoscopic instruments and "homemade" ports are being published more and more frequently.

\section{Conclusions}

The SPS nephrectomy appears possible to be used in children with a good cosmetic result. Other possible benefits, such as decrease of pain and shorter hospital stay, are still under discussion, but appear to be similar to classic laparoscopy. The use of gallbladder fixation in order to visualize the right kidney better is very beneficial, but poses additional risk of organ injury and should be performed by experienced operators.

\section{References}

1. Kaouk JH, Autorino R, Kim FJ, et al. Laparoendoscopic single-site surgery in urology: worldwide multi-institutional analysis of 1076 cases. Eur Urol 2011; 60: 998-1005.

2. Gill IS, Advincula AP, Aron M, et al. Consensus statement of the consortium for laparoendoscopic single-site surgery. Surg Endosc 2010; 24: 762-8.

3. Chłosta P, Drewa T, Obarzanowski M, et al. Do we need a cosmetic effect for radical nephrectomy? Laparoendoscopic singlesite surgery would help to answer this question. Videosurgery Miniinv 2011; 6: 1-4.

4. Koh CJ, De Filippo RE, Chang AY, et al. Laparoendoscopic singlesite nephrectomy in pediatric patients: initial clinical series of infants to adolescents. Urology 2010; 76: 1457-62.

5. Kim C, McKay K, Docimo S. Laparoscopic nephrectomy in children: systematic review of transperitoneal and retroperitoneal approaches. Urology 2009; 73: 280-4.

6. Kaouk JH, Palmer JS. Single-port laparoscopic surgery: initial experience in children for varicocelectomy BJU Int 2008; 102 : 97-9.

7. Johnsonn KC, Cha DY, DaJusta DG, et al. Pediatric single-portaccess nephrectomy for a multicystic, dysplastic kidney. J Pediatr Urol 2009; 5: 402-4

8. Ham WS, Im YJ, Jung HJ, et al. Initial experience with laparoendoscopic single-site nephrectomy and nephroureterectomy in children. Urology 2011; 77: 1204-8.

9. Tugcu V, Ilbey YO, Mutlu B, Tasci Al. Laparoendoscopic single-site surgery versus standard laparoscopic simple nephrectomy: a prospective randomized study. J Endourol 2010; 24: 1315-20.
10. Tam YH, Yin Sihoe JD, Cheung ST, et al. Single-incision laparoscopic nephrectomy and heminephroureterectomy in young children using conventional instruments: first report of initial experience. Urology 2011; 77: 711-5.

11. Raman JD, Bagrodia A, Cadeddu JA. Single-incision, umbilical laparoscopic versus conventional laparoscopic nephrectomy: a comparison of perioperative outcomes and short-term measures of convalescence. Eur Urol 2009; 55: 1198-204.

12. Bayazit Y, Aridogan IA, Abat D, et al. Pediatric transumbilical laparoendoscopic single-site nephroureterectomy: initial report. Urology 2009; 74: 1116-9.

13. Tam YH, Lee KH, Yin Sihoe JD, et al. Initial experience in children using conventional laparoscopic instruments in single-incision laparoscopic surgery. J Pediatr Surg 2010; 45: 2381-5.

Received: 25.11.2011, Revised: 7.02.2012, Accepted: 29.05.2012. 